\title{
$\beta 1$ integrin mediates an alternative survival pathway in breast cancer cells resistant to lapatinib
}

\author{
Catherine Huang ${ }^{1}$, Catherine C Park², Susan G Hilsenbeck1, Robin Ward ${ }^{1}$, Mothaffar F Rimawi ${ }^{1}$, Yen-chao Wang ${ }^{1}$,
} Jiang Shou', Mina J Bissell ${ }^{3}, C_{\text {Kent Osborne }}^{1}$ and Rachel Schiff ${ }^{1 *}$

\begin{abstract}
Introduction: The overexpression of human epidermal growth factor receptor (HER)-2 in 20\% of human breast cancers and its association with aggressive growth has led to widespread use of HER2-targeted therapies, such as trastuzumab $(T)$ and lapatinib (L). Despite the success of these drugs, their efficacy is limited in patients whose tumors demonstrate de novo or acquired resistance to treatment. The $\beta 1$ integrin resides on the membrane of the breast cancer cell, activating several elements of breast tumor progression including proliferation and survival.

Methods: We developed a panel of HER2-overexpressing cell lines resistant to $L, T$, and the potent $L T$ combination through long-term exposure and validated these models in 3D culture. Parental and L/T/LT-resistant cells were subject to HER2 and $\beta 1$ integrin inhibitors in 3D and monitored for 12 days, followed by quantification of colony number. Parallel experiments were conducted where cells were either stained for Ki-67 and Terminal deoxynucleotidyl transferase dUTP nick end labeling (TUNEL) or harvested for protein and analyzed by immunoblot. Results were subjected to statistical testing using analysis of variance and linear contrasts, followed by adjustment with the Sidak method.

Results: Using multiple cell lines including BT474 and HCC1954, we reveal that in L and LT resistance, where phosphorylation of EGFR/HER1, HER2, and HER3 are strongly inhibited, kinases downstream of $\beta 1$ integrinincluding focal adhesion kinase (FAK) and Src-are up-regulated. Blockade of $\beta 1$ by the antibody AllB2 abrogates this up-regulation and functionally achieves significant growth inhibition of $L$ and $L T$ resistant cells in 3D, without dramatically affecting the parental cells. SiRNA against $\beta 1$ as well as pharmacologic inhibition of FAK achieve the same growth inhibitory effect. In contrast, trastuzumab-resistant cells, which retain high levels of phosphorylated EGFR/HER1, HER2, and HER3, are only modestly growth-inhibited by AllB2.
\end{abstract}

Conclusions: Our data suggest that HER2 activity, which is suppressed in resistance involving $L$ but not $T$ alone, dictates whether $\beta 1$ mediates an alternative pathway driving resistance. Our findings justify clinical studies investigating the inhibition of $\beta 1$ or its downstream signaling moieties as strategies to overcome acquired $L$ and LT resistance.

\section{Introduction}

The HER signaling pathway is one of the most studied and prominent drivers of human breast cancer progression. Aberrant overexpression, activation, and dimerization of the individual members of the HER familycomprised of EGFR (Epidermal Growth Factor Receptor

\footnotetext{
* Correspondence: rschiff@bcm.edu

'Lester and Sue Smith Breast Center, Baylor College of Medicine, One Baylor Plaza, Houston, TX 77054, USA

Full list of author information is available at the end of the article
}

1)/HER1, HER2, HER3, and HER4-contribute both to aggressive tumor growth and poor patient prognosis [1]. Amidst the complexity of the HER signaling network, HER2 has received a great deal of attention due to its frequent overexpression in tumors and its status as the preferred dimerization partner of the family [2].

HER2 is amplified and/or overexpressed in about $20 \%$ of human breast cancers and is independently associated with reduced disease-free and overall survival. Two FDA-approved drugs for the treatment of HER2-

\section{Biomed Central}


overexpressing tumors are the monoclonal antibody trastuzumab, and the EGFR/HER2 tyrosine kinase inhibitor lapatinib. Each drug is effective in inducing tumor regression in some patients with metastatic disease, but remissions are temporary since resistance commonly develops [3-9]. Clinical trials are currently investigating the administration of lapatinib and trastuzumab together (LT) [8-10], which has been shown pre-clinically by our laboratory [11] and others $[12,13]$ to induce prolonged regression in breast cancer xenografts by more completely blocking downstream signals generated by various homo- and hetero-dimers of the HER family. Even this potent treatment strategy, however, gives way to resistance in many tumors. It is clear that the identification of alternative molecular pathways driving resistant growth would have important therapeutic implications.

The $\beta 1$ integrin subunit is one member of a large family of receptors that mediate the interaction between cytoskeletal elements and the extracellular matrix [14]. Each integrin is a heterodimer composed of one of 18 possible $\alpha$ subunits together with 1 of $8 \beta$ subunits. In response to laminin or fibronectin [15-21], $\beta 1$ as a mechanoreceptor is a critical mediator of breast cancer initiation and progression [20,22-24], both through its association with the HER pathway [25] and signal propagation through its downstream kinases FAK and Src [26-29]. In addition, $\beta 1$ has been linked to therapeutic resistance in multiple cancer types [30-32], its overexpression has been associated with poor overall survival in patients with early-stage breast cancer [33], and it can serve as a predictive indicator for patients with intrinsic resistance to trastuzumab [34].

Using an array of HER2-overexpressing cell lines $[14,35]$ developed to acquire resistance (Res) to lapatinib (L), trastuzumab (T), or both (LT) [36], we now report the critical role of $\beta 1$ integrin as an alternative pathway in $\mathrm{L}$ - and $\mathrm{LT}$ resistance. We demonstrate that $\mathrm{L}$ - and LTRes cells maintain strong inhibition of HER2 as well as EGFR and HER3. However, in resistant cells phosphorylation of $\beta 1$ downstream kinases FAK and Src is markedly upregulated, and this is inhibited by the $\beta 1$ antibody AIIB2. We also show that $\beta 1$ blockade by either AIIB2 or siRNA, as well as by a FAK inhibitor, significantly inhibits L- and LTRes cell growth in 3D. Parental and TRes cells, on the other hand, which retain high levels of phosphorylated EGFR, HER2, and HER3, fail to up-regulate the $\beta 1$ pathway, and respond to AIIB2 with only modest growth inhibition, suggesting that these cells rely less on the $\beta 1$ pathway than the HER pathway for growth and resistance, in contrast to LRes and LTRes cells. Most importantly parental and TRes cells in our 3D models respond to L, indicating that their growth is due to the HER pathway. Altogether our results indicate that when HER2 is inhibited, as it is in L- and LTRes breast cancer cells, $\beta 1$ signaling can operate as an alternative driver of growth.

\section{Materials and methods \\ Reagents and cell culture}

The human breast cancer cell line BT474 was purchased from American Type Culture Collection (ATCC, Manassas, VA, USA) and maintained as previously described [37]. AU565, HCC202, and HCC1954 cell lines were generously supplied by Dr. J. Gray (Berkeley, CA, USA) and grown as in [35]. Cell lines were not re-authenticated upon receipt. Three-dimensional (3D) cultures were plated as in [20] on top of growth factor-reduced $\operatorname{lrECM}$ (Trevigen, Inc., Gaithersburg, MD, USA) at a density of 3 to 6,000 cells per well of an eight-well chamberslide (LabTek, Milwaukee, WI, USA), with all relevant inhibitors added on Day 0. Media containing the inhibitors and $5 \% \operatorname{lrECM}$ were changed every 3 days and maintained for 5 to 12 days. The $\beta 1$ inhibitory antibody AIIB2 (Aragen Bioscience, Morgan Hill, CA, USA, used at $0.24 \mathrm{mg} / \mathrm{ml}$ ), FAK inhibitor PF 573228 (Tocris Bioscience, Bristol, UK, used at $1 \mu \mathrm{M}$ ), lapatinib (GlaxoSmithKline, Research Triangle Park, NC, USA, used at $1 \mu \mathrm{M}$ ), and trastuzumab (Genentech, San Francisco, CA, USA, used at $50 \mu \mathrm{g} / \mathrm{ml}$ ) were used. Control vehicles were rat IgG (Thermo Fisher Scientific, Waltham, Massachusetts, USA) and dimethyl sulfoxide (DMSO). Antibodies used include phosphorylated (p) HER2 (Y1248, Millipore, Billerica, Massachusetts, USA); pHER2 (y877), pEGFR (Y1068), EGFR, pHER3 (Y1289), HER3, pAKT (S473), AKT, pSrc (Y416), and $\beta$-actin (all from Cell Signaling Technology, Danvers, Massachusetts, USA); $\beta 1$ and FAK (BD Biosciences, Franklin Lakes, New Jersey, USA); pFAK (Y861, Biosource, Chevy Chase, Maryland, USA); and Src and HER2 (Santa Cruz Biotechnology, Santa Cruz, CA, USA).

\section{Establishment of resistant cell lines}

Resistant cell lines were developed by first exposing cells to a full dose of lapatinib $(1 \mu \mathrm{M})$ and/or trastuzumab $(50 \mu \mathrm{g} / \mathrm{ml})$ to assess the de novo resistance of each model. Drug-sensitive cell lines were subsequently cultured for 3 to 12 months in respective media supplemented with increasing concentrations of lapatinib (0.1 to $1 \mu \mathrm{M}$ ), trastuzumab ( 1 to $50 \mu \mathrm{g} / \mathrm{ml}$ ), or both, as cells developed resistance to each dosage. Resistance at each dose was assessed by comparing the growth of each resistant derivative to their parental counterparts. All resistant cells were passaged along the same schedule, twice a week, with resistant cells being split at equal or higher ratios than the parental. HCC1954 cells are de novo resistant to trastuzumab and were cultured in the continuous presence of the inhibitor both before and during experimentation. 


\section{siRNA in 2D/3D}

Cells were seeded in six-well plates at $60 \%$ confluency and subjected to two consecutive 24-hour rounds of siRNA transfection, using Hs_ITGB1_5 oligos (Qiagen, Hilden, Germany) and the Mirus Trans-IT TKO transfection reagent (Madison, WI, USA). Cells were then trypsinized and plated on top of IrECM as above. Assay medium containing 5\% lrECM, Mirus reagent, siRNA$\beta 1$, and relevant drugs was supplied to the cells and changed every three days. Cultures were propagated for 10 days and imaged. A second siRNA was utilized for data in Additional file 1 (catalog s7576, Applied Biosystems, Carlsbad, CA, USA).

\section{Protein extraction and immunoblot}

Protein extractions from 2D cultures were performed as in [35]. All panels shown were run on the same gel per cell model or, when working with overlapping markers, run on two gels in parallel. All experiments were repeated at least three times and representative results are shown.

From 3D cultures seeded in $35 \mathrm{~mm}$ dishes for at least five days, wells were washed twice with cold PBS, then replaced with PBS-EDTA (containing $5 \mathrm{mM}$ EDTA, 1 mM 0.2 M Na3VO4, 1.5 mM 0.25 M NaF, and a cocktail of protease inhibitors (Roche, Basel, Switzerland)) for 15 minutes on ice. Cultures and IrECM were then separated from the dish using a cell lifter, and contents were transferred to a conical tube for an additional 30 minutes on ice to allow full dissolution of the lrECM. Cells were then centrifuged for five minutes at 800 rpms, the supernatant aspirated, and the pellet resuspended in lysis buffer. Protein was extracted as above and subjected to Western blot analysis.

\section{Apoptosis and proliferation assays}

Apoptosis (TUNEL assay) and proliferation were assayed in-well of an eight-well chamberslide as in [20] with the following modifications: cells were fixed in $4 \%$ paraformaldehyde and Ki-67 was obtained from Vector Labs, Burlingame, CA, USA, and detected by Alexa Fluor 568 goat anti-rabbit IgG secondary antibody (Molecular Probes/Invitrogen). All slides were imaged using a Leica EL6000 inverted laser scanning confocal microscope.

\section{Quantification of 3D cultures}

All experiments were conducted in triplicate and repeated in three independent studies. Phase contrast images of the cultures in eight-well chamberslides were taken with a $4 \mathrm{x}$ objective lens, then processed and quantified by measuring colony size. A conversion factor of $1.8755 \mu \mathrm{m} /$ pixel for the $4 \mathrm{x}$ objective was determined and utilized to acquire numerical measurements for each colony imaged, with the cutoff of $50 \mu \mathrm{m}$ set as the defining value for a colony. At least nine wells per treatment group were quantified per value reported, from three independent experiments.

\section{Statistical considerations}

In vitro experiments to compare numbers of colonies formed, proliferation, and apoptosis were run in triplicate and repeated at least three times. The experiment sets had factorial designs and were analyzed using analysis of variance, which allows global analysis of all experiments in the set. All data were first transformed, by taking logarithms, in order to stabilize variances and because differential effects detected by ANOVA on the log scale can be expressed as fold changes on the raw scale, and have a natural biologic interpretation. Each "experiment" was considered a categorical blocking factor, cell line (if different cell lines were used) was a categorical factor (that is, HCC1954 and HCC1954 LTRes), and treatment (that is, IgG vs AIIB2) was a categorical factor, yielding three-way or two-way factorial designs. Analyses included "experiment" as a main effect only and included main effects and interaction between cell line and treatment, where appropriate. Specific comparisons (for example, to examine IgG vs AIIB2 within HCC1954, after adjusting for experiment) were made using linear contrasts. $P$-values for comparisons of treatments were adjusted by the Sidak method to account for multiple comparisons. For purposes of plotting, geometric means and 95\% confidence intervals were calculated by back-transforming (that is, exponentiating) model-estimated group means and 95\% confidence limits. Plots show data from three repeated experiments, executed in triplicate, combined.

\section{Results}

The $\beta 1$ integrin's downstream kinases FAK and Src are activated upon acquisition of resistance to lapatinibcontaining HER2-targeted therapies

We developed a panel of HER2-overexpressing cell lines resistant to lapatinib (LRes), trastuzumab (TRes), and the LT combination (LTRes) through long-term culturing in 2D. Immunoblot analysis of these acquired resistant cell lines revealed that phosphorylated (p) HER2 levels were mostly reduced in both LRes and LTRes BT474 (Figure 1A) and HCC1954 (Figure 1B) cells in comparison to the untreated parental, but retained high expression in acquired (BT474) or de novo (HCC1954) TRes cells. In BT474 LRes, BT474 LTRes, and HCC1954 LTRes cells, where HER2 phosphorylation was mostly inhibited, we found marked increases in levels of the $\beta 1$ integrin downstream kinases pFAK (Y861) and pSrc (Y416) (Figure 1). Interestingly, HCC1954 LRes cells, which express low levels of all markers examined, did not grow in lrECM. Additional 


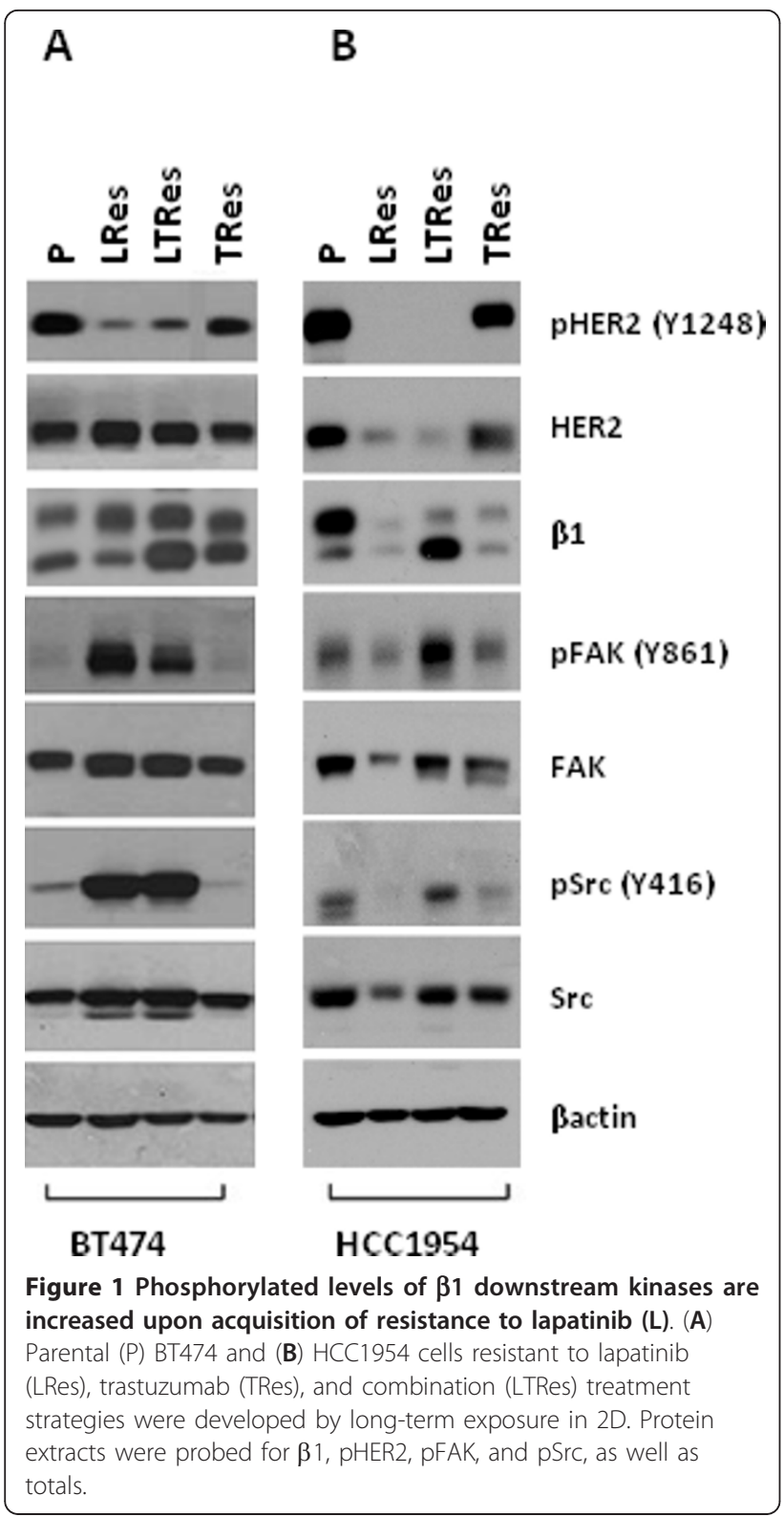

cell lines, AU565 and HCC202, yielded similar results (Additional file 2). These data led us to hypothesize that persistent inhibition of pHER2 in LRes and LTRes (but not TRes) can lead to activation of FAK and Src as an escape mechanism to circumvent HER2 blockade, and that targeting their shared upstream protein, the $\beta 1$ integrin $(\beta 1)$, could potentially circumvent the consequent resistance.

\section{$\beta 1$ inhibition overcomes $L$ resistance in ER+, HER2- amplified BT474 cells}

We next tested $\beta 1$ 's role in resistance by blocking its activity using either the inhibitory antibody AIIB2, which binds the extracellular portion of the $\beta 1$ integrin to inhibit signaling, or $\beta 1$-specific siRNA in laminin-rich extracellular matrix (lrECM) 3D culture. We used 3D culture because of its ability to recapitulate $\beta 1$ 's in vivo function, which is to mediate the communication between extracellular signals and intracellular kinases. To first validate our resistant models in 3D culture, BT474 parental and LRes cells were propagated on lrECM and assayed for growth. As expected, parental cells displayed robust growth characteristic of tumorigenic cells in 3D, but they were profoundly growthinhibited by treatment with lapatinib. LRes cells, on the other hand, grew aggressively in its presence (Figure 2A and Table 1).

We then subjected parental and LRes cells to AIIB2 treatment and found it to be highly effective in inhibiting colony growth in LRes cells $(69.7 \%$ colony growth inhibition, $P$-value $<.0001)$, while only modestly and nonsignificantly affecting parental cells $(18.8 \%$ colony growth inhibition, $P$-value $=.2498)($ Figure $2 B)$. Examination of an additional cell line, AU565 LRes, produced similar results (Additional file 3A).

To investigate the nature of the growth inhibition by AIIB2, we assessed the proliferative (Ki-67) and apoptotic (TUNEL) indices of parental and LRes BT474 cells (Figure 2D). AIIB2 inhibited proliferation only modestly and nonsignificantly in parental cells, whereas proliferation was significantly inhibited in LRes cells $(72.9 \%, P$ $<0.001)$. The degree of inhibition was significantly greater in LRes cells compared to parental cells $(P=$ 0.0018). In contrast, both parental and LRes cells underwent increased apoptosis with AIIB2 treatment (106\%, $P$ $<0.001$; 193\%, $P<0.001$, respectively), but the degree of increase was not significantly different between the cell lines $(P=0.1869$, Figure $2 C)$. Collectively these data suggest that AIIB2 elicits its differential growth inhibitory effects on LRes BT474 cells primarily by inhibiting proliferation.

\section{$\beta 1$ downstream signaling is inhibited by AllB2 and is critical for the lapatinib resistant phenotype}

We then determined how $\beta 1$ inhibition by AIIB2 could alter downstream signaling in LRes BT474 3D cultures (Figure 2D). Confirming data from Figure 1, we found that in comparison to the parental, LRes cells exhibited dramatically less pHER2 at two independent phosphorylation sites (Y877 and Y1248), while exhibiting dramatic increases in both pFAK and pSrc. The upregulation of pFAK and pSrc in LRes cells was abrogated by treatment with AIIB2. The top band of the $\beta 1$ doublet, which we believe represents posttranslational modification, was also reduced by AIIB2. Levels of PMAPK and pAKT, which reside downstream of both $\beta 1$ integrin and HER receptor signaling, were also altered. pMAPK (T202/Y204) expression was decreased in the LRes line 


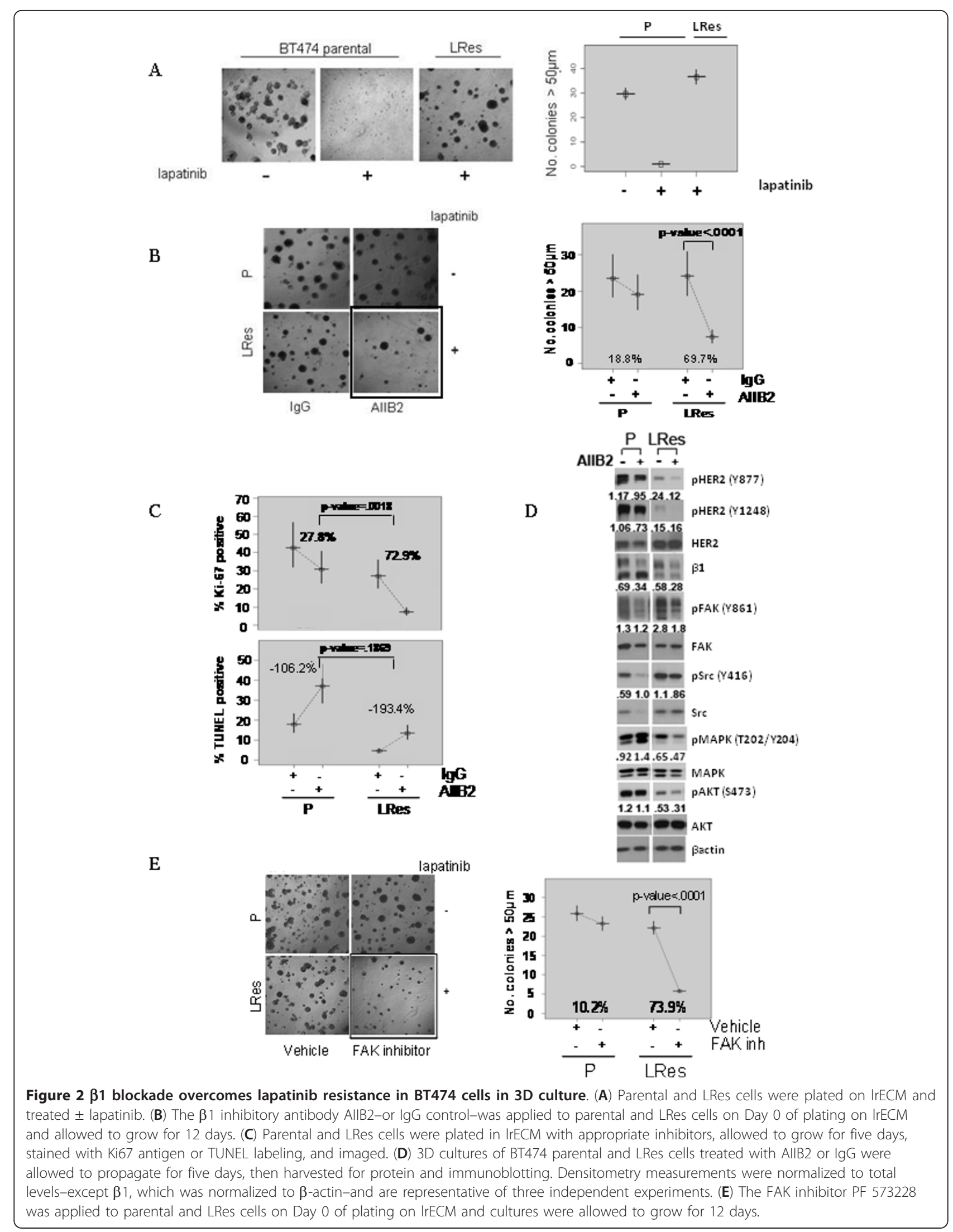


Table 1 Percent growth inhibition of cells in response to HER-targeted therapies

\begin{tabular}{lllll}
\hline Cell line & Drug $\rightarrow$ & Lapatinib & Trastuzumab & LT \\
& Derivative & \% Inhibition $\mathbf{( 9 5 \% ~ C l )}$ & \% Inhibition $(\mathbf{9 5} \% \mathbf{C l})$ & \% Inhibition (95\% Cl) \\
\hline BT474 & Parental & $100.0 \%$ & $72.1 \%(67.6 \%, 76.0 \%)$ & $100.0 \%$ \\
& Resistant & $5.7 \%(-0.3 \%, 11.4 \%)$ & $11.4 \%(5.8 \%, 16.8 \%)$ & $13.7 \%(8.6 \%, 18.6 \%)$ \\
\multirow{2}{*}{ HCC1954 } & Parental & - & $7.4 \%(3.8 \%, 10.9 \%)$ & $100.0 \%$ \\
& Resistant & - & $5.4 \%(1.4 \%, 9.2 \%)$ & $37.8 \%(32.5 \%, 42.7 \%)$ \\
\hline
\end{tabular}

An analysis of variance was performed to estimate the effect of treatment on each cell model. The estimated treatment effect and associated standard error were used to compute estimates and $95 \%$ confidence intervals, which were then back-transformed and subtracted from $100 \%$ to give the "\% inhibition."

and, importantly, further diminished with AIIB2 treatment. pAKT (S473) expression showed a similar trend (Figure 2D). These experiments, which were confirmed with AU565 cells in Additional file 3B, suggest that $\beta 1$ integrin and its downstream moieties FAK and Src, as well as MAPK and AKT, are important in BT474 LRes cells, and that neutralizing this pathway may provide a functional basis for the ability of $\beta 1$ blockade to overcome lapatinib resistance. Interestingly, both phosphoand total Src levels were reduced to undetectable levels in parental cells treated with AIIB2.

To further confirm the importance of the $\beta 1$ downstream signaling pathway in LRes cells, we utilized the FAK inhibitor PF 573228 [38]. Results mirrored our findings with AIIB2. Parental cells responded to FAK inhibition in $3 \mathrm{D}$ with a small, nonsignificant reduction in colony growth, while LRes cells were significantly more sensitive, exhibiting a $73.9 \%(P<0.0001)$ reduction in colony growth (Figure $2 \mathrm{E}$ ). These data suggest that $\beta 1$ and FAK are far more important for the LRes phenotype than for growth of parental cells and, thus, their blockade elicits more striking inhibition of LRes cells.

\section{TRes BT474 cells are inhibited by lapatinib}

Our results from Figure 1 show that pHER2 levels are reduced in both LRes and LTRes cells, suggesting that reactivation of HER2 is not involved in lapatinib resistance in these cells. We therefore further investigated the functional, differential dependence of LRes and TRes on the HER2 and the $\beta 1$ pathways. To this end, we first subjected LRes cells to trastuzumab and TRes cells to lapatinib, and compared their response to parental cells treated with each agent. As shown in Figure 3A, parental cells were markedly inhibited by both lapatinib and trastuzumab $(94.3 \%$ and $71.6 \%$, respectively, top panel; also see Table 1). In contrast, LRes cells were only modestly inhibited by trastuzumab (25\%, middle panel), an effect that was significantly weaker than the inhibitory effect achieved by trastuzumab in parental cells. TRes cells, on the other hand, were just as sensitive to lapatinib as the parental cells $(93.1 \%$ and $94.3 \%$ growth inhibition, respectively). Collectively these experiments suggest that TRes cells are dependent on the HER2 pathway, while LRes cells are not.

Since HER2 activity is affected by its homo- or heterodimerization with EGFR and HER3, we also examined whether a more global differential activation of the HER receptor layer takes place in LRes and TRes cells (Figure 3B). Phosphorylation states of all three receptors-EGFR, HER2, and HER3-were very low in LRes cells which were actively proliferating, suggesting that these cells are driven by a pathway distinct from the HER family. TRes cells, in contrast, had relatively high levels of pEGFR, pHER2, and pHER3 similar to the parental cells, suggesting that the HER pathway is active and explaining why these cells are inhibited by lapatinib (Figure 3A). These data were confirmed with the AU565 model as shown in Additional file 3B.

Having shown that TRes cells are dependent on the HER pathway while LRes cells are not, we next investigated the differential dependence of these resistant clones on $\beta 1$ integrin. We expanded our studies to include clones resistant to $\mathrm{L}+\mathrm{T}$ treatment, which our data (Figure 1) suggest are similar to LRes. Upon validating the LTRes phenotype in 3D culture (Figure 4A and Table 1), we assessed the response of LTRes BT474 cells to AIIB2. Similarly to LRes, the LTRes cells also exhibited significant growth inhibition in response to AIIB2 $(63.8 \%, P<0.0001$, and $80 \%, P<0.0001$, respectively, Figure $4 B$ ). On the other hand, TRes cells-which retain high HER receptor activity and do not display robust upregulation of $\beta 1$ integrin signaling (Figure 1)were only slightly and nonsignificantly inhibited by AIIB2 at a level comparable to parental cells $(27.8 \%$ and $24.3 \%$, respectively, $P=.87$, Figure $4 \mathrm{~B}$ ).

To further establish the specificity of $\beta 1$ inhibition, an siRNA approach was employed. $\beta 1$ protein expression knockdown in 2D as well as in $3 \mathrm{D}$ cultures over 9 days was confirmed (Figure 4C, top). The siRNA was then applied in $3 \mathrm{D}$ to confirm our findings with AIIB2. As before, siRNA- $\beta 1$ inhibited parental BT474 cell growth only modestly, but it almost completely inhibited both LRes and LTRes growth (Figure 4C, bottom) and induced apoptosis (Additional file 4). These findings 
A
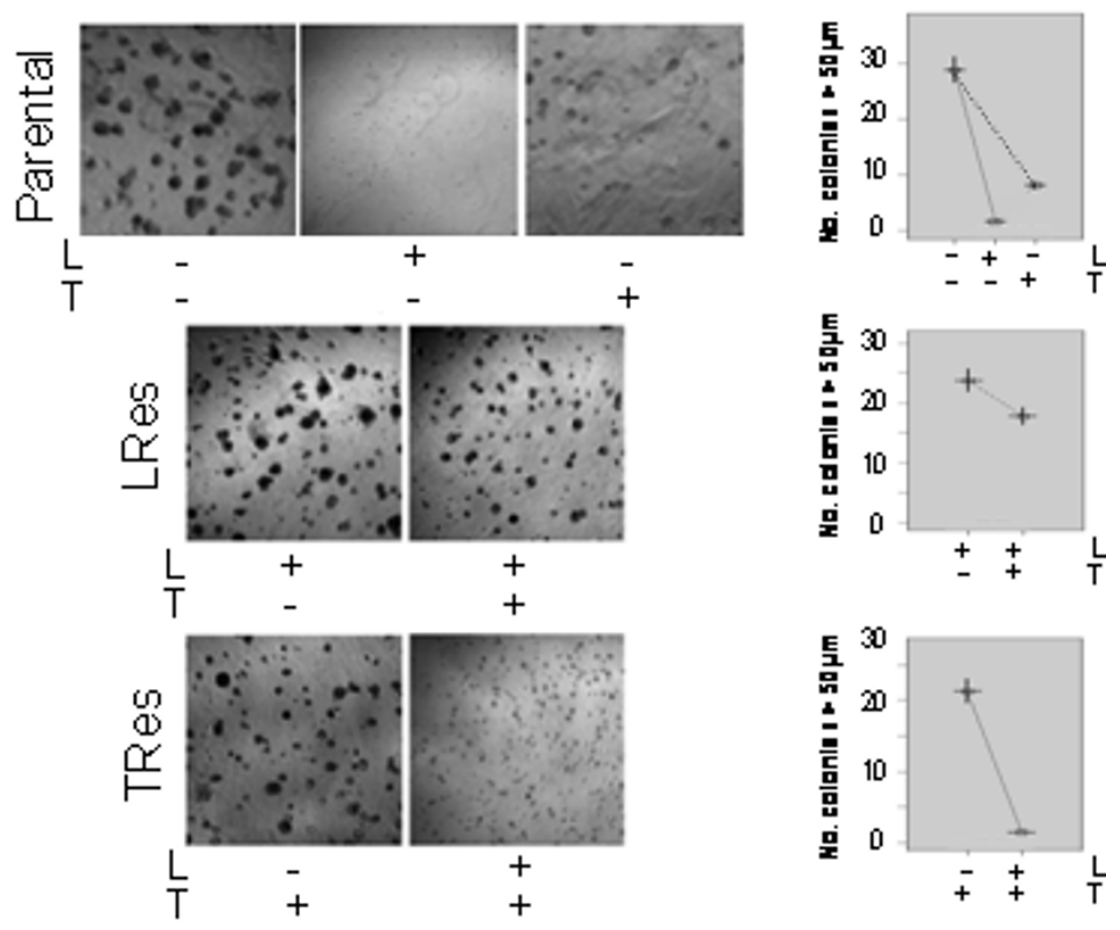

B

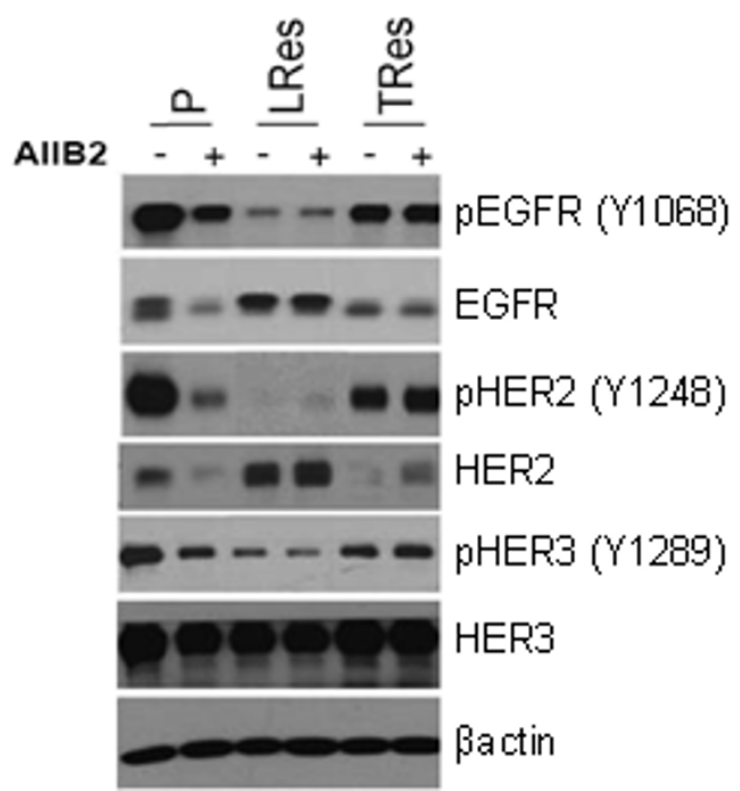

Figure 3 HER2 and the $\beta 1$ pathway play alternate roles in resistance to lapatinib-containing regimens, in comparison to trastuzumab (A) Parental, LRes, and TRes cells were plated on IrECM in the presence of lapatinib and/or trastuzumab and assayed for response. (B) 3D cultures of parental, LRes, and TRes BT474 cells were harvested for protein and probed for phosphorylated and total HER receptors. 
A

B

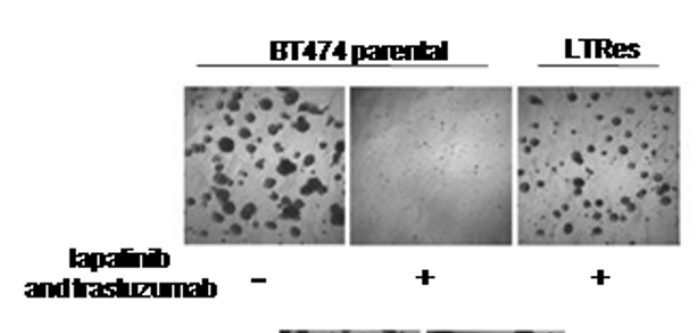

P

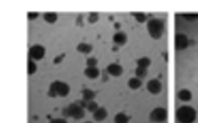

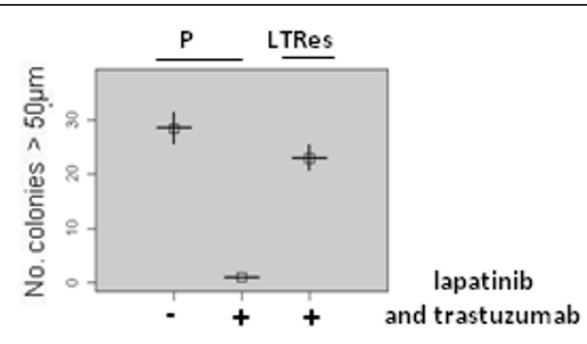

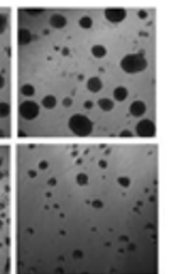

LTRes

LRes

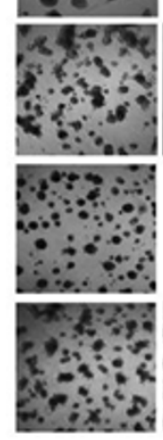

IgG AllB2

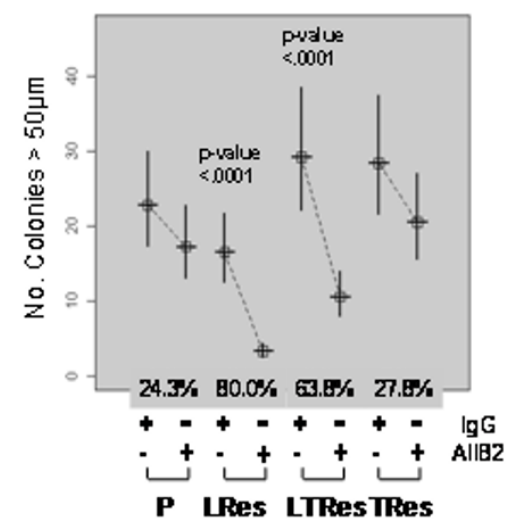

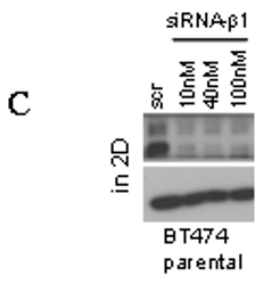

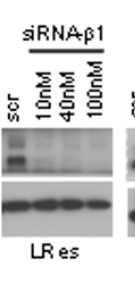

胃NAB1

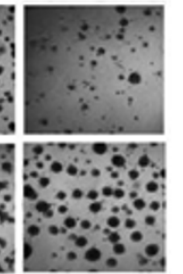

TRes

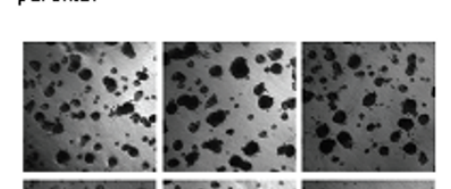

LRes
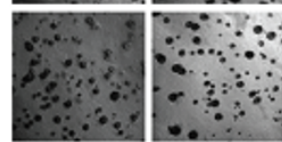

LTRes

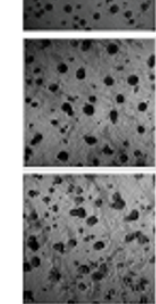

Ctrl
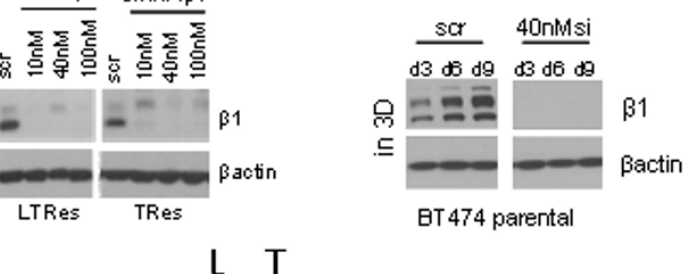

L $\quad \mathrm{T}$

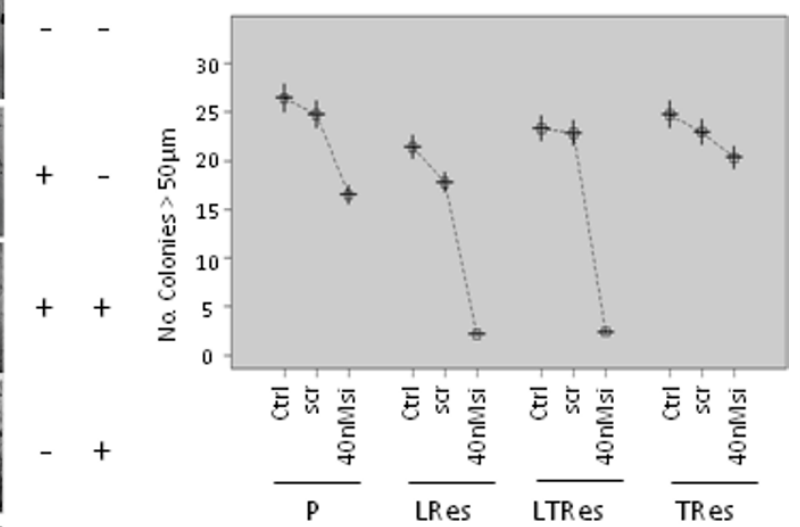

Figure 4 ק1 inhibition impedes colony grow of BT474 LRes and LTRes cells, but not parental or TRes. (A) Parental, LRes, LTRes, and TRes cells were plated in IrECM, subjected to HER2 and/or $\beta 1$ inhibitors on Day 0, and propagated for 10 to 12 days. (B) The Hs_ITGB1_5 siRNA was validated both in 2D and 3D (top), transfected at $40 \mathrm{nMsi}$ into parental, LRes, LTRes, and TRes cells, which were then grown on IrECM for 10 days (bottom). 
were confirmed with a second independent siRNA sequence (Additional file $1 \mathrm{~A}$ ). Altogether, the $\beta 1$ inhibition studies using AIIB2 and siRNA- $\beta 1$ indicate that LRes and LTRes cells are significantly more dependent on the $\beta 1$ pathway than TRes cells or their parental counterparts, and are thus more sensitive to $\beta 1$ blockade.

\section{$\beta 1$ inhibition overcomes LT resistance in ER-, HER2- amplified HCC1954 cells}

The LT combination, which conveys a more complete blockade of the HER receptor layer than HER2-targeted monotherapy, is currently in clinical trials in both the adjuvant and neo-adjuvant settings. To extend our findings in LTRes BT474 cells to another cell line, we chose HCC1954 cells, which are ER-, HER2-amplified, and highly aggressive [39-41], and validated this model on lrECM (Figure 5A and Table 1). Similar to BT474 cells, parental cells were only modestly inhibited by AIIB2 (31.0\% reduction in colony number, $P<0.0001$, Figure $5 \mathrm{~B})$. In contrast, LTRes cells were almost completely growth-inhibited by blocking $\beta 1$ integrin (92.6\% reduction, $P$-value $<.0001$, Figure $5 \mathrm{~B}$ ), a reduction significantly greater than parental cells $(P<0.0001)$. Examination of an additional ER-, HER2-amplified LTRes cell lineHCC202-corroborated the functional and differential efficacy of AIIB2 on the resistant LTRes derivative (Additional file 3C).

We also examined the effect of $\beta 1$ blockade on proliferation and apoptosis in HCC1954 cells (Figure 5C). In contrast to BT474 cells, we found that AIIB2 significantly inhibited proliferation in both parental $(53.1 \%, P$ $<.0001)$ and LTRes cells $(65.2 \%, P<.0001)$. Induction of apoptosis, however, was markedly greater $(P<.0001)$ in LTRes compared to parental cells $(209.9 \%, P<.0001$ vs. $53.6 \%, P<.0001$, respectively; also see Additional file 4). Thus, although the basal levels of both proliferation and apoptosis varied between parental and LTRes cells, AIIB2 exerted a highly significant differential effect on the induction of apoptosis. These findings indicate that $\beta 1$ integrin blockade with AIIB2, while reducing 3D colony formation in both BT474 and HCC1954 cell lines, has a predominantly cytostatic effect in BT474. LRes cells but a cytotoxic effect in HCC1954 LTRes cells.

We next examined how $\beta 1$ blockade exerted its functional effects on HCC1954 cell growth and survival by surveying $\beta 1$ signaling intermediates (Figure 6A). Levels of pHER2 at two separate sites were, as in BT474 LRes cells, very low in LTRes cells. Levels of pFAK and pSrc were dramatically higher upon acquisition of resistance to LT therapy, and they were markedly reduced by treatment with AIIB2. AIIB2 affected the levels of $\beta 1$ and pFAK in parental cells as well, but the significantly weaker functional effect of the $\beta 1$ inhibitor on $3 \mathrm{D}$ culture growth, juxtaposed with its complete growth inhibition of LTRes cells, suggests that $\beta 1$ signaling plays a more critical role in the growth of LTRes cells where HER2 signaling remains blocked, than it does in parental cells. Finally, pAKT expression is low upon development of LTRes (confirmed in HCC202 cells, Additional file 3D), and it was further decreased by treatment with AIIB2. AKT can be activated by $\beta 1$ integrin signaling, and this further reduction in pAKT by AIIB2 may also contribute to the growth inhibitory effects in these cells. pMAPK levels, on the other hand, were not inhibited by AIIB2 in HCC1954 LTRes cultures (Figure 6A).

Treatment with the FAK inhibitor PF 573228 confirmed the importance of the $\beta 1 /$ FAK signaling axis in HCC1954 LTRes cells (Figure 6B). Inhibition of growth of LTRes cells (92.1\%) was significantly greater than in the parental cells $(31.9 \%)$ (the difference between groups, $P<0.0001)$. Similar to our results with AIIB2, these experiments suggest that $\beta 1 /$ FAK signaling is critical for the LTRes phenotype.

\section{TRes HCC1954 cells are only modestly responsive to AlIB2, in contrast to LTRes cells}

Similar to BT474 cells, levels of pEGFR, pHER2, and pHER3 in HCC1954 LTRes cells were undetectable in comparison to the high levels found in parental or TRes cells, which are dependent on HER2 for proliferation and survival (Figure 6C). Similar data were also observed in HCC202 cells (Additional file 3D). Interestingly, total HER3 expression was also decreased in HCC1954 LTRes cells, an effect not seen in the BT474 model.

As above, we wanted to determine the efficacy of the $\beta 1$ inhibitory antibody in the various HCC1954 derivatives. Of note, we attempted to assay LRes HCC1954 cells in 3D but could not grow them on lrECM. We thus reassessed parental HCC1954 cells alongside the LTRes and long term-cultured TRes derivative cells (Table 1) in 3D, where HCC1954 cells are de novo resistant to trastuzumab due to a mutation in PI3K. We found that TRes, like parental cells, were only modestly but significantly responsive to AIIB2 (32.0\% inhibition, $P$ $<.0001$ and $31.0 \%, P<.0001$, respectively), whereas LTRes cells, as above, were $92.5 \%$ inhibited $(P<.0001$, Figure 7A). The degree of inhibition between parental and TRes cells was not significant $(P=.52)$ but was indeed significant between parental and LTRes cells $(P$ $<.0001)$.

Using a genetic approach, we confirmed knockdown of $\beta 1$ protein levels post transfection with siRNA- $\beta 1$ or a scrambled sequence, before plating on IrECM (Figure 7B, top). Cultures were imaged after 10 days (Figure 7B, bottom) and protein extracted every 3 days to confirm sustained $\beta 1$ knockdown (Figure $7 \mathrm{~B}$, top). A second 
A

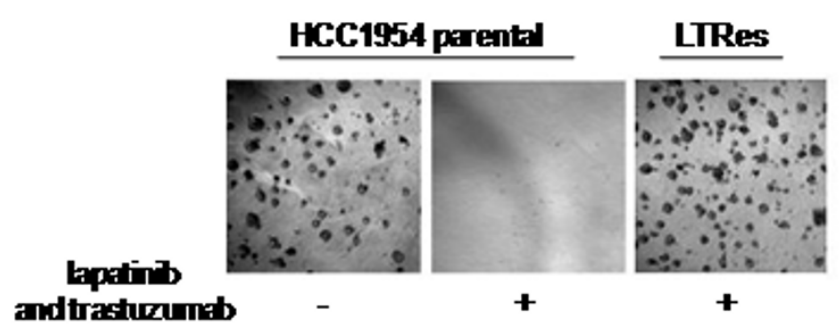

B

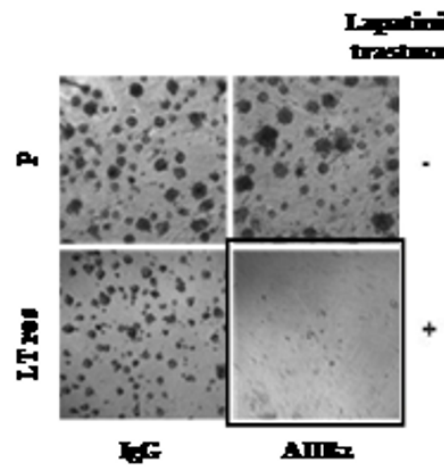

C

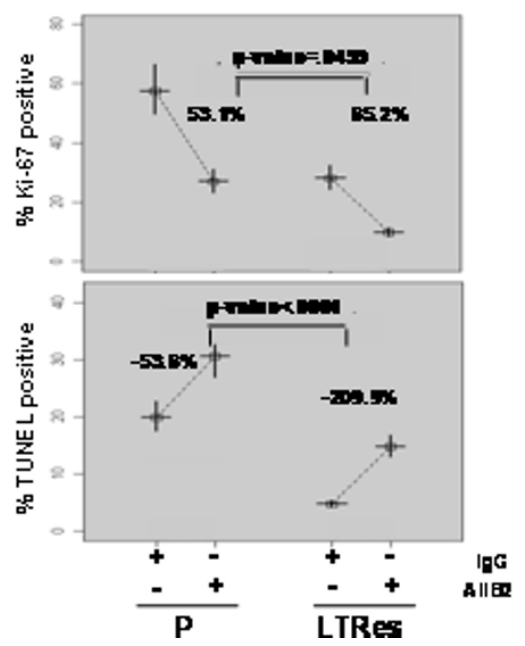

Figure 5 $\beta 1$ inhibition overcomes resistance to potent LT therapy in HCC1954 cells. (A) HCC1954 parental and LTRes were plated on IrECM in the presence of LT and assayed for growth response. (B) Cells were plated on IrECM, subjected to either AllB2 or lgG control, and imaged after 12 days. (C) Cultures were grown in the presence of appropriate inhibitors for five days and immunostained with Ki67 or TUNEL reagent as before. 


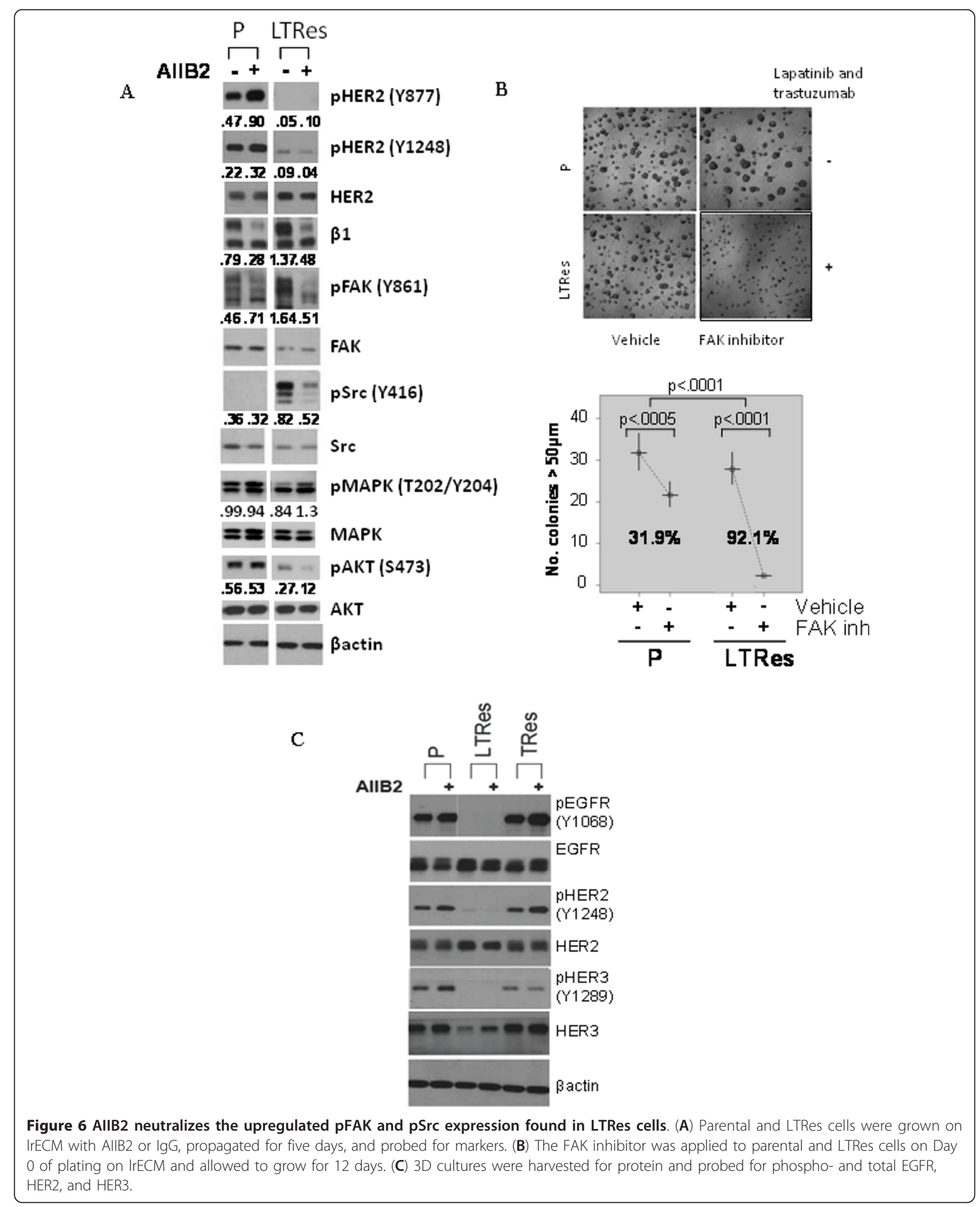




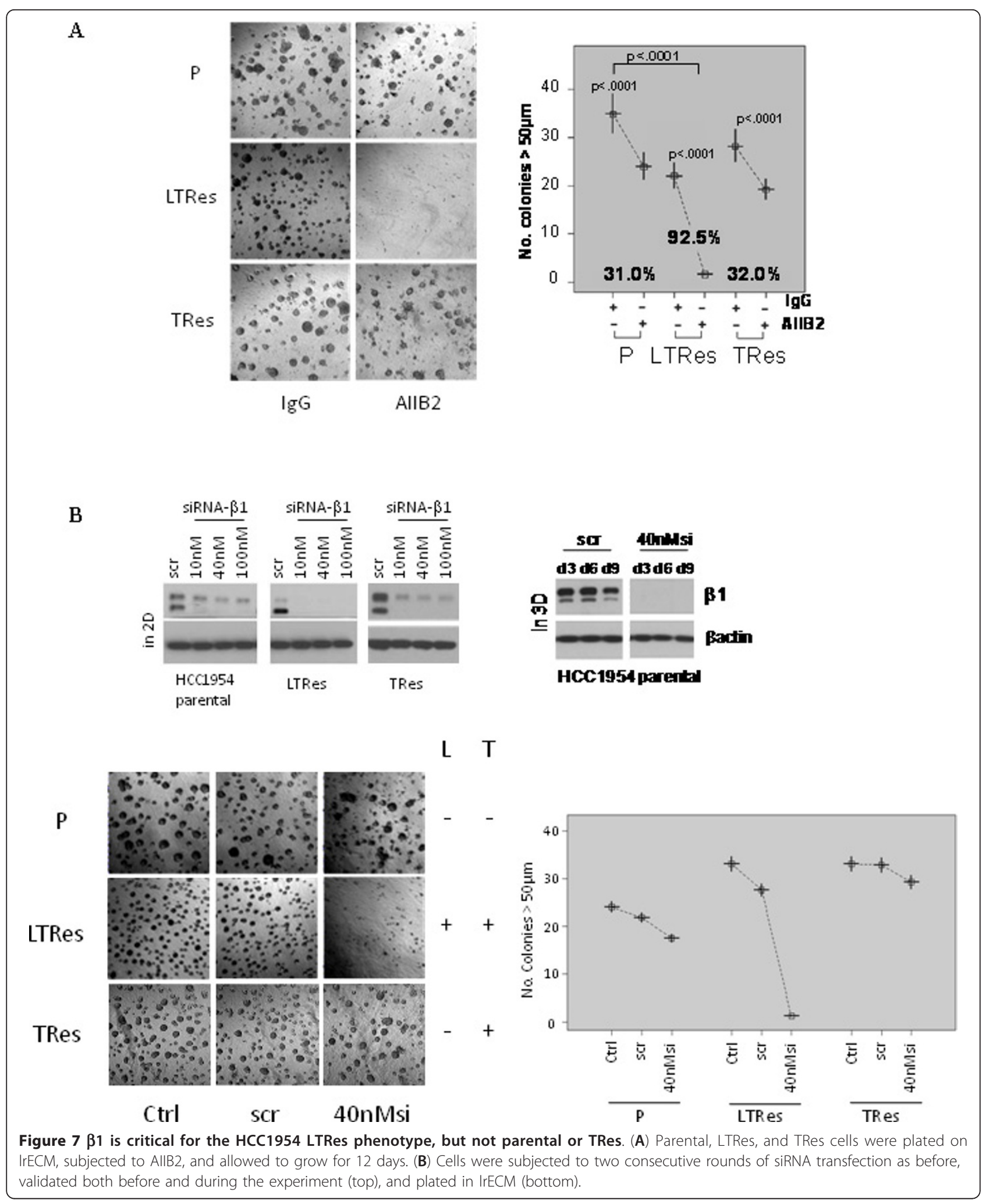


independent sequence (Additional file 1B) yielded similar results. siRNA- $\beta 1$ inhibited growth of the parental and TRes cells only modestly, but inhibited LTRes cell growth completely (Figure 7B). Collectively these results suggest that HCC1954 LTRes cells are more dependent on $\beta 1$ signaling than either the parental or TRes cells, and confirm with a second cell model that resistance to lapatinib-containing regimens-where HER2 remains inhibited-is exceedingly more responsive to $\beta 1$ inhibition than in TRes, where HER signaling remains active.

\section{Discussion}

Multiple mechanisms of trastuzumab resistance have been proposed in both preclinical and clinical studies [42], such as incomplete blockade of the HER receptor layer $[36,37,43]$, PTEN loss, or activating mutations in PI3K, but the causes of acquired resistance to lapatinib are less clear [9]. The microenvironment has been shown to influence various cell survival and proliferation pathways $[33,44,45]$. Here, we provide evidence that the $\beta 1$ integrin, a receptor that transmits signals from the microenvironment, plays an important role as an escape pathway in acquired resistance to $L$ and LT, where HER signaling remains strongly inhibited.

We now demonstrate that HER2-overexpressing LRes and LTRes cells, while maintaining strong inhibition of phosphorylation of EGFR, HER2, and HER3, exhibit marked up-regulation of $\beta 1$ signaling by activation of its downstream kinases, FAK and Src. Enhancement of pFAK and pSrc levels is specifically greater in LRes and LTRes than in TRes cells. Several studies independently investigating mechanisms of resistance to $\mathrm{L}$ and $\mathrm{T}$ have shown that LRes is associated with continued inhibition of HER receptors [46] or the HER pathway [12,47], while TRes is associated with reactivation of the HER pathway $[48,49]$. We found $\beta 1$ signaling components to be more prominently up-regulated in L- and LTRes cells compared to TRes cells. As in the prior studies, LRes and LTRes cells have low levels of phosphorylated HER2, EGFR, and HER3, indicating that the HER pathway is still shut down.

Using multiple HER2-amplified cell line models, some ER+ and some ER-, and their therapeutically resistant derivatives grown in $\operatorname{lrECM}$, we found that parental cells were only modestly responsive to $\beta 1$ inhibition by the antibody AIIB2, by siRNA- $\beta 1$, or by FAK inhibition with PF573228. LRes and LTRes cells, in contrast, were significantly growth-inhibited, suggesting a greater dependence of these resistant cells on $\beta 1$ signaling than their parental counterparts. In further elucidating the mechanism of action underlying $\beta 1$ inhibition, we found that AIIB2 modulated $\beta 1$ expression and effectively suppressed both pFAK and pSrc, as well as pAKT, in both parental and LRes or
LTRes derivatives. It is possible that the $\beta 1$ pathway also promotes growth and survival in parental cells, but our data suggest that HER2 remains the primary driver (Figure 3A). It is interesting to note that while up-regulation of $\beta 1$ protein in resistant cells was not always observed (Figures 1, 2D), $\beta 1$ pathway activation could potentially be achieved several ways, which include the release of ECM ligands, integrin clustering, and/or the activation of downstream markers. Our studies showed that LRes and LTRes cells exhibit increases in the $\beta 1$ downstream markers pFAK and pSrc when compared to the parental cells, and that inhibition of $\beta 1$ is able to reduce the high levels of phosphorylated FAK and Src found in cells resistant to lapatinib. These observations suggest that some HER2overexpressing breast cancer cells become more dependent on $\beta 1$ signaling as they acquire resistance to $L$, a potent HER receptor inhibitor, and that blocking this escape pathway can restore inhibition of tumor growth.

Interestingly, we found that in cells resistant to L-containing regimens, blocking $\beta 1$ can elicit an apoptotic or predominantly anti-proliferative response, depending on the cell line studied. The molecular mechanisms responsible for this differential response to $\beta 1$ inhibition are not clear, although it is interesting to note that the inhibition of BT474 cell proliferation coincides with a reduction in pMAPK, while inhibition of HCC1954 LTRes cells is associated with increased apoptosis and a marked reduction in pAKT.

In comparing acquired LRes or LTRes to single-agent TRes, we found that $\beta 1$ inhibition by AIIB2 was significantly and specifically effective in those cells resistant to lapatinib-containing treatments. Importantly, even doubling the dose of lapatinib in LRes and LTRes cells did not inhibit growth of either cell line in 3D (Additional File 5), further suggesting that growth of these L-resistant lines is independent of HER2. Under these conditions, our data suggest that the $\beta 1$ pathway compensates at least in part for the blockade of HER signaling. Both acquired (BT474) and de novo (HCC1954) TRes cells, on the other hand, maintain their dependence on HER2, as evidenced by their sensitivity to lapatinib [13,48] (Figure $3 \mathrm{~A}$ ) and the high levels of pEGFR, pHER2, and pHER3 (Figures 3B, 6C).

There have been recent publications suggesting a role for $\beta 1$ integrin in intrinsic trastuzumab resistance $[34,44]$. Our studies focused on acquired resistant cell lines developed through chronic exposure. As such, our data do not dispute these reports. We also find that growth of TRes cells is modestly inhibited by blocking $\beta 1$. Our results suggest, however, that $\beta 1$ integrin signaling is a much more prominent escape pathway for HER2-amplified tumors treated by L or LT, than by $\mathrm{T}$ 
alone. Studies of tumor tissue from patients with acquired resistance to $\mathrm{L}$ or $\mathrm{T}$ are required to learn whether these preclinical observations have clinical relevance.

\section{Conclusions}

Although multiple mechanisms likely underlie and contribute to lapatinib resistance, our data suggest that $\beta 1$ integrin signaling is a promising therapeutic target to block and thereby inhibit growth of resistant tumors in patients.

\section{Additional material}

Additional file 1: A second siRNA sequence applied to BT474 and HCC1954 cells in 3D IrECM confirms that LRes and LTRes cells depend more critically on $\beta 1$ than their parental counterparts Double, consecutive rounds of siRNA transfection at $40 \mathrm{nM}$ were executed and cells plated directly onto IrECM for 10 days, followed by quantification.

Additional file 2: Phosphorylated levels of the $\beta 1$ downstream kinases FAK and Src are increased in additional HER2overexpressing cell line models upon acquisition of resistance to lapatinib (L)-containing HER-targeted therapies. (A) Parental (P) AU565 and (B) HCC202 cells resistant to lapatinib (LRes) and combination (LTRes) treatment strategies were developed by long-term exposure in 2D. Protein extracts were probed for $\beta 1$, pHER2, pFAK, and pSrc, as well as totals.

Additional file 3: $\beta 1$ blockade overcomes resistance to lapatinibcontaining regimens in AU565 and $\mathrm{HCC} 202$ cells and abrogates upregulated pFAK and pSrc expression. (A) and (C) Cells were propagated in $3 \mathrm{D}$ IrECM and treated with respective inhibitors and/or AllB2. Statistical analysis was conducted as in Figure 2. (B and $\mathbf{D}$, left) 3D extracts of AU565 cells exhibit upregulated protein expression of $\beta 1$, pFAK, and pSrc upon acquisition of resistance to lapatinib. These effects are neutralized upon application of the $\beta 1$ inhibitory antibody AllB2. Expression of phosphorylated levels of MAPK and AKT are decreased in LRes cells in comparison to their parental counterparts. (B and $\mathbf{D}$, right) The HER receptor layer is effectively inhibited in L- and LT-Res cells but remains active in both parental and TRes cells.

Additional file 4: Genetic blockade of $\beta 1$ by siRNA in BT474 and HCC1954 cells induces apoptosis. Cells were transfected with siRNA, plated onto IrECM, propagated for five days, then harvested using the TUNEL assay as in Figure $2 \mathrm{C}$.

Additional file 5: Doubling the dose of lapatinib in cells resistant to lapatinib-containing regimens does not dramatically affect growth. BT474 LRes and HCC1954 LTRes cells were first primed in 2D with $2 \mu \mathrm{M}$ lapatinib (twice the usual dose) for five days. Cells were then plated onto IrECM, propagated for 12 days, and quantified.

\section{Abbreviations}

EGFR: Epidermal Growth Factor Receptor; ER: Estrogen Receptor; T: trastuzumab; L: lapatinib; IrECM: laminin-rich extracellular matrix; p: phosphorylated; Res: resistant

\section{Acknowledgements}

This work was funded by Susan G. Komen Race for the Cure (BCTR0708226), Breast Cancer Specialized Program of Research Excellence grant (SPORE) P50 CA058183 from the National Cancer Institute, the Entertainment Industry Foundation (EIF)/ Lee Jeans Translational Breast Cancer Research Program, the Stand Up to Cancer Breast Cancer Program, and the Breast Cancer Research Foundation (BCRF). We acknowledge support from $\mathrm{NIH}$ 1R01CA124891-01 and American Cancer Society RSG-07-1110-01-CCE to CP.

\section{Author details}

'Lester and Sue Smith Breast Center, Baylor College of Medicine, One Baylor Plaza, Houston, TX 77054, USA. ²Department of Radiation Oncology, University of California San Francisco, 1600 Divisadero Street, MZ BIdg R, San Francisco, CA 94143, USA. ${ }^{3}$ Department of Cancer \& DNA Damage Responses, Lawrence Berkeley National Laboratories, One Cyclotron Road, Berkeley, CA 94720, USA.

\section{Authors' contributions}

$\mathrm{CH}$ conducted the molecular studies presented in this manuscript, including 3D culturing, application of pharmacological and genetic approaches to inhibit $\beta 1$, immunostaining, protein extraction, and immunoblotting. CP, MR, and MJB contributed substantially to the conception and design of the data. SH performed all statistical analyses necessary for each molecular study. RW and YW developed the lapatinib, trastuzumab, and LT resistant cell lines through long term exposure of parental cells to each inhibitor or set of inhibitors. CKO and RS conceived of the study and its design and implementation. All authors read and approved the final manuscript.

\section{Competing interests}

The authors declare that they have no competing interests.

Received: 9 January 2011 Revised: 24 May 2011

Accepted: 31 August 2011 Published: 31 August 2011

\section{References}

1. Citri A, Yarden Y: EGF-ERBB signalling: towards the systems level. Nat Rev Mol Cell Biol 2006, 7:505-516.

2. Yarden Y: Biology of HER2 and its importance in breast cancer. Oncology 2001, 61(Suppl 2):1-13.

3. Bartsch R, Wenzel C, Zielinski CC, Steger GG: HER-2-positive breast cancer: hope beyond trastuzumab. BioDrugs 2007, 21:69-77.

4. Browne BC, O'Brien N, Duffy MJ, Crown J, O'Donovan N: HER-2 signaling and inhibition in breast cancer. Curr Cancer Drug Targets 2009, 9:419-438.

5. Nahta R, Esteva FJ: Trastuzumab: triumphs and tribulations. Oncogene 2007, 26:3637-3643.

6. Hudis CA: Trastuzumab-mechanism of action and use in clinical practice. N Engl J Med 2007, 357:39-51.

7. Valabrega G, Montemurro F, Aglietta M: Trastuzumab: mechanism of action, resistance and future perspectives in HER2-overexpressing breast cancer. Ann Oncol 2007, 18:977-984.

8. Spector NL, Blackwell KL: Understanding the mechanisms behind trastuzumab therapy for human epidermal growth factor receptor 2positive breast cancer. J Clin Oncol 2009, 27:5838-5847.

9. Esteva FJ, Yu D, Hung MC, Hortobagyi GN: Molecular predictors of response to trastuzumab and lapatinib in breast cancer. Nat Rev Clin Oncol 2010, 7:98-107.

10. Blackwell KL, Burstein HG, Sledge GW, Stein S, Ellis C, Casey M, Baselga J, O'Shaughnessy J: Updated survival analysis of a randomized study of lapatinib alone or in combination with trastuzumab in women with HER2-positive metastatic breast cancer progressing on trastuzumab therapy. Cancer Res 2009, 69(Suppl 3):61.

11. Rimawi MF, Weiss HL, Bhatia P, Chamness G, Elledge RM: EGFR expression in breast cancer: association with biologic phenotype, prognosis, and resistance to adjuvant therapy. Journal of Clinical Oncology 24(June 20 Supplement):513, 2006 ASCO Annual Meeting Proceedings Part I.

12. Scaltriti M, Verma C, Guzman M, Jimenez J, Parra JL, Pedersen K, Smith DJ, Landolfi S, Ramon y Cajal S, Arribas J, Baselga J: Lapatinib, a HER2 tyrosine kinase inhibitor, induces stabilization and accumulation of HER2 and potentiates trastuzumab-dependent cell cytotoxicity. Oncogene 2009, 28:803-814.

13. Konecny GE, Pegram MD, Venkatesan N, Finn R, Yang G, Rahmeh M, Untch M, Rusnak DW, Spehar G, Mullin RJ, Keith BR, Gilmer TM, Berger M, Podratz KC, Slamon DJ: Activity of the dual kinase inhibitor lapatinib (GW572016) against HER-2-overexpressing and trastuzumab-treated breast cancer cells. Cancer Res 2006, 66:1630-1639.

14. Kenny PA, Lee GY, Myers CA, Neve RM, Semeiks JR, Spellman PT, Lorenz K, Lee EH, Barcellos-Hoff MH, Petersen OW, Gray JW, Bissell MJ: The morphologies of breast cancer cell lines in three-dimensional assays correlate with their profiles of gene expression. Mol Oncol 2007, 1:84-96. 
15. Miranti CK, Brugge JS: Sensing the environment: a historical perspective on integrin signal transduction. Nat Cell Biol 2002, 4:E83-90.

16. Giancotti FG, Ruoslahti E: Integrin signaling. Science 1999, 285:1028-1032.

17. Chrenek MA, Wong P, Weaver VM: Tumour-stromal interactions. Integrins and cell adhesions as modulators of mammary cell survival and transformation. Breast Cancer Res 2001, 3:224-229.

18. Mercurio AM, Bachelder RE, Chung J, O'Connor KL, Rabinovitz I, Shaw LM, Tani T: Integrin laminin receptors and breast carcinoma progression. J Mammary Gland Biol Neoplasia 2001, 6:299-309.

19. Weaver VM, Petersen OW, Wang F, Larabell CA, Briand P, Damsky C, Bissell MJ: Reversion of the malignant phenotype of human breast cells in three-dimensional culture and in vivo by integrin blocking antibodies. J Cell Biol 1997, 137:231-245.

20. Park CC, Zhang H, Pallavicini M, Gray JW, Baehner F, Park CJ, Bissell MJ: Beta1 integrin inhibitory antibody induces apoptosis of breast cancer cells, inhibits growth, and distinguishes malignant from normal phenotype in three dimensional cultures and in vivo. Cancer Res 2006, 66:1526-1535.

21. Nam JM, Onodera Y, Bissell MJ, Park CC: Breast cancer cells in threedimensional culture display an enhanced radioresponse after coordinate targeting of integrin alpha5beta 1 and fibronectin. Cancer Res 70:5238-5248.

22. Cui $X$, Lee AV: Regulatory nodes that integrate and coordinate signaling as potential targets for breast cancer therapy. Clin Cancer Res 2004, 10:396S-401S.

23. Shaw LM: Integrin function in breast carcinoma progression. J Mammary Gland Biol Neoplasia 1999, 4:367-376.

24. Cordes N, Park CC: beta1 integrin as a molecular therapeutic target. Int J Radiat Biol 2007, 83:753-760.

25. Wang F, Weaver VM, Petersen OW, Larabell CA, Dedhar S, Briand P, Lupu R, Bissell MJ: Reciprocal interactions between beta1-integrin and epidermal growth factor receptor in three-dimensional basement membrane breast cultures: a different perspective in epithelial biology. Proc Natl Acad Sci USA 1998, 95:14821-14826.

26. Planas-Silva MD, Bruggeman RD, Grenko RT, Stanley Smith J: Role of c-Src and focal adhesion kinase in progression and metastasis of estrogen receptor-positive breast cancer. Biochem Biophys Res Commun 2006, 341:73-81.

27. Hiscox S, Morgan L, Green TP, Barrow D, Gee J, Nicholson Rl: Elevated Src activity promotes cellular invasion and motility in tamoxifen resistant breast cancer cells. Breast Cancer Res Treat 2006, 97:263-274.

28. Hui AY, Meens JA, Schick C, Organ SL, Qiao H, Tremblay EA, Schaeffer E, Uniyal S, Chan BM, Elliott BE: Src and FAK mediate cell-matrix adhesiondependent activation of Met during transformation of breast epithelial cells. J Cell Biochem 2009, 107:1168-1181.

29. Mitra SK, Schlaepfer DD: Integrin-regulated FAK-Src signaling in normal and cancer cells. Curr Opin Cell Biol 2006, 18:516-523.

30. Aoudjit F, Vuori K: Integrin signaling inhibits paclitaxel-induced apoptosis in breast cancer cells. Oncogene 2001, 20:4995-5004.

31. Damiano JS, Cress AE, Hazlehurst LA, Shtil AA, Dalton WS: Cell adhesion mediated drug resistance (CAM-DR): role of integrins and resistance to apoptosis in human myeloma cell lines. Blood 1999, 93:1658-1667.

32. Hodkinson PS, Mackinnon AC, Sethi T: Extracellular matrix regulation of drug resistance in small-cell lung cancer. Int J Radiat Biol 2007, 83:733-741.

33. Park CC, Zhang HJ, Yao ES, Park CJ, Bissell MJ: Beta1 integrin inhibition dramatically enhances radiotherapy efficacy in human breast cancer xenografts. Cancer Res 2008, 68:4398-4405.

34. Lesniak D, Xu Y, Deschenes J, Lai R, Thoms J, Murray D, Gosh S, Mackey JR, Sabri S, Abdulkarim B: \{beta\}1-integrin circumvents the antiproliferative effects of trastuzumab in human epidermal growth factor receptor-2positive breast cancer. Cancer Res 2009, 69:8620-8628.

35. Neve RM, Chin K, Fridlyand J, Yeh J, Baehner FL, Fevr T, Clark L, Bayani N, Coppe JP, Tong F, Speed T, Spellman PT, DeVries S, Lapuk A, Wang NJ, Kuo WL, Stilwell JL, Pinkel D, Albertson DG, Waldman FM, McCormick F, Dickson RB, Johnson MD, Lippman M, Ethier S, Gazdar A, Gray JW: A collection of breast cancer cell lines for the study of functionally distinct cancer subtypes. Cancer Cell 2006, 10:515-527.

36. Wang Y-C, Ward R, Rimawi M, Osborne C, Schiff R: Different mechanisms for required resistance to trastuzumab and lapatinib in HER2 positive breast cancers: role of ER and HER2 reactivation. San Antonio Breast Cancer Symposium 2009, Abstract 708.

37. Arpino G, Gutierrez C, Weiss H, Rimawi M, Massarweh S, Bharwani L, De Placido S, Osborne CK, Schiff R: Treatment of human epidermal growth factor receptor 2-overexpressing breast cancer xenografts with multiagent HER-targeted therapy. J Natl Cancer Inst 2007, 99:694-705.

38. Slack-Davis JK, Martin KH, Tilghman RW, Iwanicki M, Ung EJ, Autry C, Luzzio MJ, Cooper B, Kath JC, Roberts WG, Parsons JT: Cellular characterization of a novel focal adhesion kinase inhibitor. J Biol Chem 2007, 282:14845-14852.

39. Alles MC, Gardiner-Garden M, Nott DJ, Wang Y, Foekens JA, Sutherland RL, Musgrove EA, Ormandy CJ: Meta-analysis and gene set enrichment relative to ER status reveal elevated activity of MYC and E2F in the "basal" breast cancer subgroup. PLoS One 2009, 4:e4710.

40. Marty B, Maire V, Gravier E, Rigaill G, Vincent-Salomon A, Kappler M, Lebigot I, Djelti F, Tourdes A, Gestraud P, Hupe P, Barillot E, Cruzalequi F, Tucker GC, Stern MH, Thiery JP, Hickman JA, Dubois T: Frequent PTEN genomic alterations and activated phosphatidylinositol 3-kinase pathway in basal-like breast cancer cells. Breast Cancer Res 2008, 10:R101.

41. Herschkowitz Jl, He X, Fan C, Perou CM: The functional loss of the retinoblastoma tumour suppressor is a common event in basal-like and luminal B breast carcinomas. Breast Cancer Res 2008, 10:R75.

42. Shattuck DL, Miller JK, Carraway KL, Sweeney C: Met receptor contributes to trastuzumab resistance of Her2-overexpressing breast cancer cells. Cancer Res 2008, 68:1471-1477.

43. Ritter CA, Perez-Torres M, Rinehart C, Guix M, Dugger T, Engelman JA, Arteaga $\mathrm{CL}$ : Human breast cancer cells selected for resistance to trastuzumab in vivo overexpress epidermal growth factor receptor and ErbB ligands and remain dependent on the ErbB receptor network. Clin Cancer Res 2007, 13:4909-4919.

44. Weigelt B, Lo AT, Park CC, Gray JW, Bissell MJ: HER2 signaling pathway activation and response of breast cancer cells to HER2-targeting agents is dependent strongly on the 3D microenvironment. Breast Cancer Res Treat 2010, 122:35-43.

45. Guo W, Pylayeva Y, Pepe A, Yoshioka T, Muller WJ, Inghirami G, Giancotti FG: Beta 4 integrin amplifies ErbB2 signaling to promote mammary tumorigenesis. Cell 2006, 126:489-502.

46. Xia W, Bacus S, Hegde P, Husain I, Strum J, Liu L, Paulazzo G, Lyass L, Trusk P, Hill J, Harris J, Spector NL: A model of acquired autoresistance to a potent ErbB2 tyrosine kinase inhibitor and a therapeutic strategy to prevent its onset in breast cancer. Proc Natl Acad Sci USA 2006, 103:7795-7800.

47. Liu L, Greger J, Shi H, Liu Y, Greshock J, Annan R, Halsey W, Sathe GM, Martin AM, Gilmer TM: Novel mechanism of lapatinib resistance in HER2positive breast tumor cells: activation of AXL. Cancer Res 2009, 69:6871-6878.

48. Nahta R, Yuan LX, Du Y, Esteva FJ: Lapatinib induces apoptosis in trastuzumab-resistant breast cancer cells: effects on insulin-like growth factor I signaling. Mol Cancer Ther 2007, 6:667-674.

49. Dokmanovic M, Hirsch DS, Shen Y, Wu WJ: Rac1 contributes to trastuzumab resistance of breast cancer cells: Rac1 as a potential therapeutic target for the treatment of trastuzumab-resistant breast cancer. Mol Cancer Ther 2009, 8:1557-1569.

\section{doi: $10.1186 / \mathrm{bcr} 2936$}

Cite this article as: Huang et al:: $\beta 1$ integrin mediates an alternative survival pathway in breast cancer cells resistant to lapatinib. Breast Cancer Research 2011 13:R84. 